\title{
THIRD DISCUSSION SESSION
}

(Thursday Afternoon; 7 September, 1972)

(following the review paper by Boyarchuk)

\section{Chairman: G. LARSSON-LEANDER}

Larsson-Leander: Thank you, Dr. Smak, for giving us this account of Boyarchuk's paper. The paper is now open for discussion.

Underhill: The question with expanding envelopes is: how do you detect them? If you look at the resonance lines in the far ultra-violet spectra of O-type and early B-type supergiants, you see displaced absorption lines, corresponding to velocities of $-1000 \mathrm{~km} \mathrm{~s}^{-1}$, and we say that matter is leaving the star. No feature in the ordinary spectral region gives you that assurance - yet now we know that those stars have expanding envelopes. The question is not whether an expanding envelope is a rare thing, but when can we detect one? Perhaps all stars have expanding envelopes, in the sense that all are shedding material to some extent. I wonder, does this affect the interstellar matter? We say that stars are formed from the interstellar matter and that they deplete it. Are they creating as much as they are taking away? Again, as Huang asked yesterday: where does the energy come from? Following the arguments of Lucy and Solomon (1970), we think that radiation has a lot to do with it. Is there any other force that could be used to give a radial acceleration outward? This is just what Huang was talking about yesterday. How do you get an outward velocity for a shell, or for any material, whether it comes off in the plane of an orbit, or a plane of rotation?

Smak: If I understand properly, there is a sequence of questions one should answer. First, is the rotational velocity of Be stars sufficiently high to associate the formation of the disks with rotational break-up? If so, the second question will be: are those B-type stars without emission that are also rotating rapidly, rotating too slowly to form disks? The third question, if that is not the case, is: if the rotation is incapable of forming disks, is the Lucy-Solomon mechanism efficient enough in the case of the Be stars, but not in the case of the ordinary B-type stars? That question, I think, should be answered by both observers and theoreticians.

Underhill: I think that the Lucy-Solomon mechanism, radiation pressure driving the material off and giving it that needed outward acceleration, is the key factor. After all, some rapidly rotating B-type stars are known to show Be spectra for a while - then the emission disappears again. There seems to be no particular pattern in the visibility of the shell - it appears roughly every ten or fifteen years. I think this must be intimately connected with some extra source of radiation pressure, just below the surface of the star. Once a B-type star has begun to move off the main sequence, it will contract a bit and then start burning hydrogen in a shell. If the shell is near enough to the surface, there may be enough radiation to give that little extra 
spurt of radiation pressure which emits a shell of gas for a while. This gradually drifts away, or falls back into the star, and then, ten or fifteen years later, the star gives itself a shake and gives another little burst. Mendoza (1958) has suggested that Be stars are just a bit above the main sequence, and that would fit with the idea that they are beginning shell-burning of hydrogen.

Huang: That's exactly the mechanism mentioned in my review paper, but I didn't have time to discuss it yesterday. Main-sequence stars are not exactly unstable, according to Lucy and Solomon. They become unstable only if they are rotating rapidly.

De Groot: But why, then, are there other rapidly rotating B-type stars which, as far as we know, are not going through an emission-line phase?

Underhill: They presumably have not advanced far enough off the main sequence to have shell-burning sufficiently near the surface that the radiation pressure gets out enough to give the needed outward acceleration.

Plavec: I'm afraid that this idea of having hydrogen shell-burning very close to the surface may be misleading because actually the hydrogen-burning shell is formed very deep inside the star, very far from its surface, and the hydrogen-burning extends over a period of the order of $10^{5} \mathrm{yrs}$ or something like that so it would be rather surprising that there would be such large changes in 10 or 15 yrs. As Dr. Huang mentioned, redistribution of angular momentum might be a better explanation than any serious change in the hydrogen-burning shell.

Hutchings: Did Dr. de Groot say that there are rapidly rotating stars that don't show emission lines? Are these rotating at break-up velocity? Is it not true to say that $\mathrm{Be}$ stars, rotational $\mathrm{Be}$ stars, are rotaing at maximum velocity?

De Groot: They are a little bit slower.

Underhill: Not all Be stars are rapidly rotating! There are so-called pole-on stars, and there are some stars that people believe have naturally sharp-lined spectra and show emissions.

Hutchings: I was just going to say that maybe this should be pointed out. Nevertheless, pole-on stars may still rotate. There are also B-type supergiants which are called Be but do not rotate rapidly. They are much more luminous than ordinary Be stars, and their surface gravity is low. Mass loss from them is a different matter altogether.

Underhill: It has even been suggested that the main-sequence Be stars with sharplined spectra can be divided into two groups (Schild, 1966). One group appears to contain intrinsically rapid rotators, while the other consists of slow rotators.

Hutchings: About Be stars lying off the main sequence, Roxburgh and Strittmatter (1965) said that this can be explained by an aspect effect. If you look at a rotating star equator-on, it is gravity-darkened and hence appears to lie off the main sequence. So the effect need not be an evolutionary one.

Bolton: There has been an idea around for some time that the Wolf-Rayet stars are remnants of mass-exchange in close binaries (Paczyński, 1967). There is a class of OB stars discovered and defined by Walborn (1971) in the spectra of which the ab- 
sorption lines show carbon and nitrogen anomalies somewhat analogous to those found in the Wolf-Rayet spectra. Because a process like that suggested for the WolfRayet stars might have operated on these stars, Mr. Lars Rogers and myself have begun to obtain spectra of those that are accessible from the Northern Hemisphere. We now have perhaps two dozen spectra of about eight of these stars and all but two of these can tentatively be identified as double-line binaries. The other two show indications of expanding atmospheres. One of these stars, HD 235679, is particularly interesting. It is a Be star with double emission with a $V / R$ of 3 or 4 to 1 . The emission is above the continuum at least down to $\lambda 3889$. The absorption lines are as sharp as any I've seen among early B-type stars, but on the Grant comparator the weak lines look clearly doubled. There is no indication of a shell spectrum or of any broad underlying absorptions.

Underhill: Not every stellar shell has a rapidly rotating star underlying.

Bolton: But there are definitely two components to the emission, a $V$ and an $R$ component. That's what is exceptional about this star. The Balmer-line emission is visible at least to $\lambda 3889$ on one plate and $\lambda 3797$ on another, so the Balmer decrement is quite small.

Underhill: This is an unusual object, and I don't think we should assign it to any class.

Bolton: I agree, but I think that its spectral anomalies suggest a possible relationship with the Wolf-Rayet stars, and at least suggest the possibility that mass exchange is going on, or has gone on.

Underhill: It can't be a Wolf-Rayet star; you never see double emission lines in the spectrum of a Wolf-Rayet star - they're just broad and rounded.

Bolton: I am not suggesting that the star is a Wolf-Rayet star. The carbon-nitrogen anomalies are in the absorption line spectrum. But the possible relationship to the Wolf-Rayet stars seems obvious to me.

Underhill: The fact that you have nitrogen lines excited rather than carbon is very probably correlated with the level of the temperature in the outer gas - for which you must consider non-LTE physics, radiation-dominated physics. It's very hard to make generalized statements, but if the temperature is above $100000 \mathrm{~K}$ and the density is about $10^{9}$ atoms $\mathrm{cm}^{-3}$, the nitrogen ions will dominate. Below $100000 \mathrm{~K}$, but above $50000 \mathrm{~K}$, the carbon ions are dominant. I am only discussing this qualitatively, but keep in mind that you must not make generalizations based on LTE calculations.

De Groot: This suggestion by Paczyński, that a Wolf-Rayet star comes into being after mass exchange in a binary, is very attractive. I like it very much. But it does not give a complete answer to how you create the Wolf-Rayet phenomenon, because it does not explain how the Wolf-Rayet spectra of the nuclei of planetary nebulae appear. So, it may contribute to an understanding of Wolf-Rayet stars, but not to the more general phenomenon and there must be other reasons for this phenomenon as well.

Underhill: Well, you can follow that up by looking at the spectrum of Sco X-1, the optical spectrum of Sco X-1. If you read the descriptions given in the literature, 
it sounds very much like the spectrum of a Wolf-Rayet star, except the lines are not as broad as in the spectra of average population I Wolf-Rayet stars. I'm sure nobody is going to call Sco X-1 a Wolf-Rayet star. It just comes back to the fact that if you have a plasma of density around $10^{9}$ to $10^{11}$ and an electron temperature around $10^{5}$, you're going to get a Wolf-Rayet spectrum, and any combination of events that gives you those densities and those temperatures, gives you a Wolf-Rayet atmosphere.

Larsson-Leander: Thank you. Any more comments on this topic? Has Dr. Smak any comments on the $U$ Gem stars which are perhaps always close binaries? Do any of us have any remarks on the symbiotic stars?

Underhill: So far as the symbiotic stars are concerned, I sometimes wonder whether it is completely beyond imagination that you're looking at the central part of a red giant that has been shedding matter and is getting down to the last remnants of its outer atmosphere and every once in a while you see through a little hole to the hot blue central core. The hole closes over and you see the atmosphere again. Is a symbiotic object really only one star - a long-period variable in the last gasps of emitting its outer atsmophere?

Hutchings: This would seem unlikely for AG Peg which has a very well-kept period of about $814 \mathrm{~d}$. You wouldn't expect a star to open up, so that you can have a look inside it, fairly regularly like that.

Plavec: I'm going to talk on symbiotic stars in my review, so I don't want to spend too much time on them now. I think it is now very reasonably established that stars like AG Peg and Z And are binaries in which the blue component flares up (or, if you don't like that term, brightens up). In the case of Z And, a flare is rather short; in AG Peg it takes several years, but when it eventually decreases again, you can see very clearly the $\mathrm{M}$-type spectrum of the other component. So it seems to me, at least, that the binary nature of this object is fairly well established.

Thackeray: AR Pav is a remarkable case of 'multiple symbiosis'. Its spectrum consists of a hot nebular spectrum with forbidden lines, indicating clouds of very low densities; a cooler nebular spectrum, a supergiant $\mathrm{F}$ shell absorption, varying in intensity, and TiO seen only near mid-eclipse. Mrs. Mayall's value for the period of $605 \mathrm{~d}$ (Mayall, 1937) has been maintained closely for some 50 cycles. There is an eclipse of some $2^{\mathrm{m}} \cdot 5$, but there are large fluctuations of $\pm 1^{\mathrm{m}}$. about a mean light-curve.

Sahade obtained some spectra in 1948 showing a P Cyg contour to the $\mathrm{H}$ lines with absorptions displaced $-100 \mathrm{~km} \mathrm{~s}^{-1}$. These contours have changed and Radcliffe spectra over 20 yrs show the rather sharp $H$ lines cut into by an absorption. The model proposed in my paper of 1959 has had to be revised in one respect since radial velocities are now available.

The sharp $\mathrm{H}$ absorptions remain at more or less constant velocity, shifted by $-22 \mathrm{~km} \mathrm{~s}^{-1}$ relative to the centre of gravity of the system, while the emissions oscillate back and forth in the period of $605 \mathrm{~d}$. Thus, there is an expanding $\mathrm{H}$ cloud surrounding the whole system. The permitted He emissions vary with semi-amplitude $K=13 \mathrm{~km} \mathrm{~s}^{-1}$, and yield a mass function $0.13 M_{\odot}$. The supergiant $\mathrm{F}$ absorption, only occasionally seen, also varies cyclically and in phase with the $\mathrm{He}$ emission with large 
scatter and $K \sim 26 \mathrm{~km} \mathrm{~s}^{-1}$. Surprisingly, the forbidden emission velocities ([O III], [Ne III]) seem to vary in opposite phase as though associated at least statistically with the secondary.

However, new evidence that the secondary is in fact an M-type star, responsible for the TiO seen at mid-eclipse, has been provided by observations by Dr. Ian Glass at the recent 1972 eclipse. He found 'no change' in the infrared ( $J, H, K, L$ bands). It will be of interest to see if a secondary eclipse becomes visible in these infrared bands at phase $0 . P$.

Leung: Are those large variations in brightness more or less semi-periodic or absolutely random?

Thackeray: Well, there has been no systematic photometry apart from Mrs. Mayall's light curve based on Harvard plates covering 40 yrs. We have a little photometry at Radcliffe, but this obviously should be followed up in detail with regular photometry at some place.

Larsson-Leander: Perhaps we could have a comment on the $\mathrm{T}$ Tau stars. I myself found Walker's (1972) interpretation of the ultra-violet excess and the blue continuum rather fascinating. Any more comments on Boyarchuk's paper?

De Groot: If I remember rightly, Dr. Smak said that in the paper it was mentioned that P Cyg stars show many similarities to the Be stars. I don't know exactly what Boyarchuk meant, but I don't see this clearly, and I'm afraid that this is a type of confusion about what stars we should call P Cyg stars. I do not think that all stars that have a P Cyg profile somewhere in the whole range of the spectrum should be called P Cyg stars, but this term should be reserved for those stars whose spectra show many more lines of various elements with this profile. Otherwise, we would mix into one pot various types of stars which really have quite different characteristics.

Larsson-Leander: We are free to leave 'expanding atmospheres' now, and to take topics left over from this morning. Mr. Bopp wants to talk about work by himself and Mr. Moffett on YY Gem.

Bopp: The eclipsing, double-lined spectroscopic binary YY Gem (Castor C) has been known to be a remarkable system since the work of Joy and Sanford (1926). The system consists of two late-type dwarfs, of spectral type dMle, with the Balmer lines and $\mathrm{Ca}$ II $\mathrm{H}$ and $\mathrm{K}$ visible in emission. The period, from von Gent's (1931) photometric study is 0.8142822 . Joy and Sanford's initial study showed marked variation in the relative strengths of the two emission components, with the red emission component being consistently stronger than the blue. Struve's special interest in this system resulted in three separate spectroscopic investigations of this system. The first (Struve et al., 1950) found equal intensities for the Balmer and Ca II components, with little, if any, evidence for variations. Two later investigations (Struve, 1952; Struve and Zebergs, 1959) found only small, irregular emission-line variations on a rapid time-scale.

As a further complication, the photoelectric investigations by Kron (1952) revealed the presence of secondary fluctuations in light that were periodic with the rotation, but temporary in duration. Kron attributed these variations to non-uniform illumi- 
nation of the stellar surfaces ('star spots'). Most recently, YY Gem has been shown to be a flare star by Moffett and Bopp (1971).

Approximately forty coudé spectra at a dispersion of $18 \AA \mathrm{mm}^{-1}$ have been obtained with the McDonald Observatory $207-\mathrm{cm}$ (82-inch) reflector during the periods 1971, February 6-17 and 1971, November 27 to 1972, February 1. The Balmer and Ca II emissions are generally of approximately equal strength, but plate No. 7154, taken on 1971 , February 10 , at phase 0.715 , is radically different. On this plate, the blue shifted component is markedly stronger than the red (Figure 1). Emission of $\mathrm{Si} \mathrm{I}$ at $\lambda 3905$ is prominent; this emission is not seen in the normal spectrum of YY Gem, but is known to be strongly enhanced in solar flares. Clearly a flare occurred, or was in progress during the hour-long exposure. However, on this spectrum the Balmer emission is not double, but triple (Figure 2). Midway between the two stellar

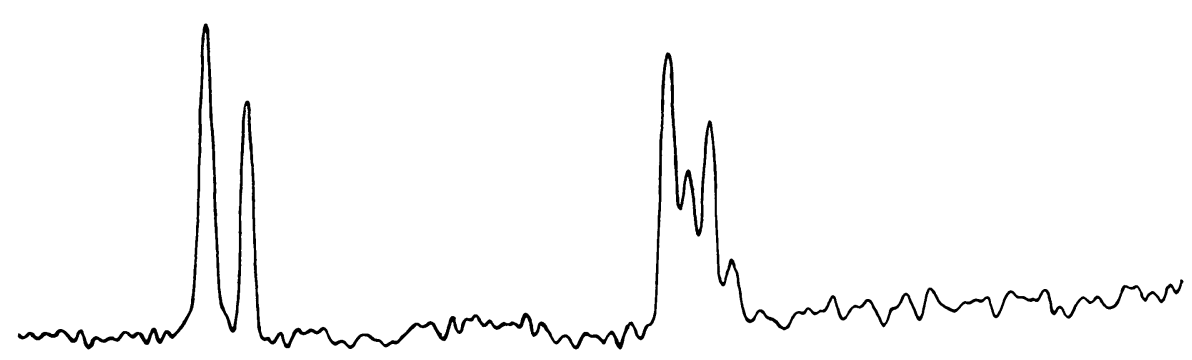

Fig. 1. Smoothed density tracing of the $\mathrm{Ca}$ II $\mathrm{H}$ and $\mathrm{K}$ region; the blue shifted component of $\mathrm{H}, \mathrm{K}$, and $\mathrm{H} \varepsilon$ is noticeably enhanced.

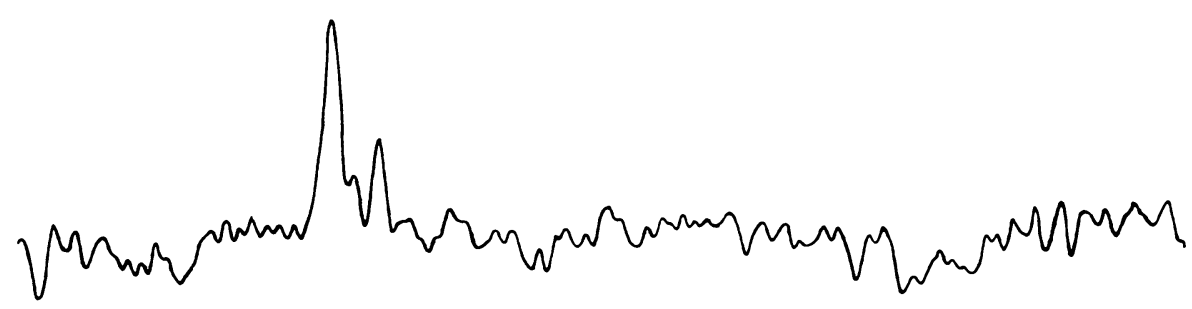

Fig. 2. Smoothed density tracing of the region containing $\mathrm{H} \gamma$. Note enhanced blue-shifted component, central emission, and quiescent red-shifted component. 
components is a weaker, narrow third component. Radial velocity measurements show this central component to have a velocity coincident with the $\gamma$-velocity of YY Gem. Another coudé plate, taken two hours later, shows the blue-ward stellar component still slightly enhanced, but the central emission is no longer visible. The central component is definitely not visible when the stars are completely quiescent.

We conclude that the central emission feature is caused by material at the central Lagrangian point of the system, and is excited into emission by flare activity on one of the components. This configuration would not, of course, be stable; the material, if a permanent feature of the system, would have to be replenished. This could occur either via explosive ejection of material during flares or by a gradual outflow of chromospheric material. Regarding the latter possibility, Struve and Zebergs noted a systematic difference of absorption-line and emission-line radial velocities in the spectrum of YY Gem, and ascribed it to a possible outflow of material. Possible connections between this material and the occurrence of flare activity are, at the moment, highly speculative. Many hypotheses concerning flare stars and circumstellar material have been discussed, however (Greenstein, 1950; M. Johnson, 1953; LortetZuckerman, 1965; Evans, 1971). Till now there has been no evidence for any association of UV Cet-type flare stars with circumstellar matter.

Leung: Are all your observations photometric?

Bopp: We have many simultaneous spectroscopic and photometric observations of YY Gem. Unfortunately, the interesting plate was obtained in 1971, February, before we started our simultaneous programme, so we do not know how bright this particular flare was.

Leung: I have some observations of YY Gem, too, and I wondered if our observing runs overlapped.

Fracastoro: Could you remind us what the light curve of YY Gem looks like?

Moffett: The light curve of YY Gem, obtained by Kron, shows nothing strange except the secondary light variations which he attributed to spots on one of the components. The two stars are practically identical in terms of mass, luminosity, etc. and the orbit is nearly circular. I would like to make a few additional comments: The word 'flare' has been used many times during this symposium to describe a wide variety of features and, as Dr. Batten has pointed out, the term 'flare' does not have a unique meaning. In discussing YY Gem, we use the word 'flare' to describe the eruptive events as observed on the UV Cet class of stars. The flares observed in YY Gem appear to be almost identical to flares occurring on other UV Cet stars. The flare shapes and colours seem to be characteristic of the UV Cet class, but the frequency of occurrence exhibits a somewhat different behaviour. In 1971, when flares were first detected on YY Gem, we observed one flare event every four hours, but in 1972 the flare activity decreased to about one every ten to twelve hours of observing. This does not occur in the other UV Cet stars; rather, their mean frequency of flare activity remains very constant year to year. The change in flare frequency on YY Gem most probably is related to the secondary light variations which Kron and Struve found some years and which were absent in others. 
Smak: Have you made any estimates as to whether the expansion velocity of $7 \mathrm{~km} \mathrm{~s}^{-1}$ is sufficient to lift the matter from the surface of the star?

Bopp: The velocity of escape from an M0 dwarf is about the same as that from the Sun, so for material to be ejected from the system would require a velocity of $650 \mathrm{~km} \mathrm{~s}^{-1}$, if we disregard the effects of the companion star. If there is a slow leakage of material some source of acceleration would be required - I do not yet know what it is. I might mention that in the ultra-violet spectrum of YY Gem, around $3200 \AA$, there are emission lines of Fe II, which are also seen in the spectra of red giants. In these latter, the lines have been interpreted as arising from an extended atmosphere or corona.

Larsson-Leander: If there are no more comments on that topic, we will ask Dr. Robinson to speak on high-speed photometry of Z Cam.

Robinson: I have been making high-speed photometric observations of $\mathrm{Z}$ Cam, using Nather's photometer on the 82-inch Struve telescope at McDonald Observatory. The time resolution of the observations, which were made without filters, is between one and five seconds. The observations show that a model similar to that developed by Smak (1971) and by Warner and Nather (1971) for U Gem also explains the major features of Z Cam. The period of Z Cam is seven hours; the primary is a G1 star, on the main sequence, that fills its Roche lobe and transfers mass to a white dwarf of about solar mass. The white dwarf is surrounded by an optically thick disk of gas, and is invisible except during eruptions. A bright spot is formed where the stream hits the disk and contributes at least $20 \%$, and probably more than $50 \%$, of the white

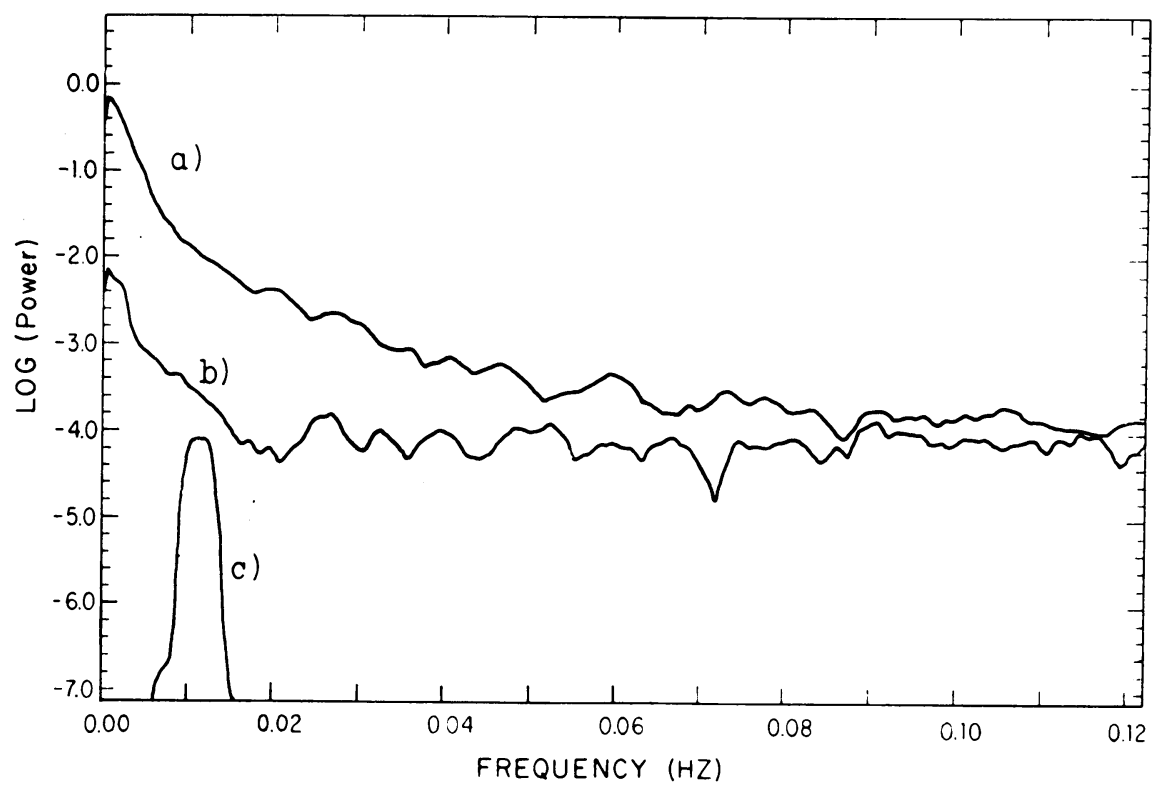

Fig. 3. Power spectrum of light variations of $\mathrm{Z}$ Cam (see text for explanation). 
light of the system during minimum. The brightness of the spot varies dramatically, thus giving rise to the flickering in the light curve.

I have made a time-series spectral analysis of my light curves of $Z$ Cam. The upper curve in Figure 3 shows the power spectrum of a typical light curve near minimum light. The middle curve is the power spectrum of the 'light curve' of a comparable constant star. Both curves have been smoothed, and the lower curve is the spectral window resulting from the smoothing. For frequencies less than about $0.09 \mathrm{~Hz}$ (period $>11 \mathrm{~s}$ ) the power spectrum is a measure of the flickering in Z Cam. It is impossible to interpret this directly, but progress can be made by looking for changes in the power spectrum, from night to night. The distribution of the power does not change, but the total power does - the curve moves up and down without changing

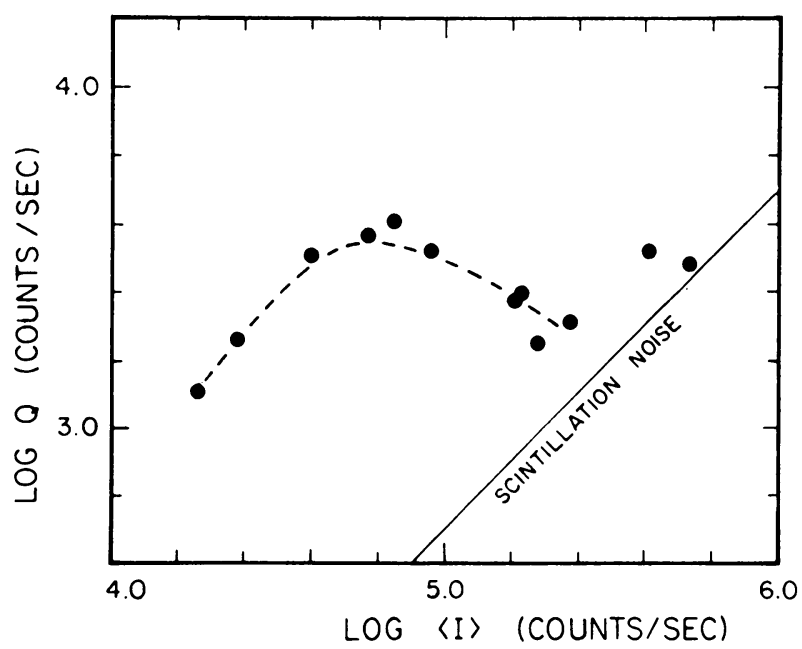

Fig. 4. Plot of $\log Q$ against $\log \langle I\rangle$ (quantities are defined in text).

shape. The frequency distribution is constant over the entire cycle of eruption. It is difficult to believe that the G-type star could erupt without affecting the process of mass transfer. For this and other reasons, I assume that the eruptions are in the white dwarf.

The light curve of $\mathrm{Z}$ Cam can be approximated by a cubic polynomial. Because of the preceding result, changes in the light curve can be described by a single parameter. I have chosen $Q$, the square root of the variance of the light curve about the polynomial: $Q$ is proportional to the total power. Figure 4 shows a plot of $\log Q$ versus $\log \langle I\rangle$ (where $I$ is the mean brightness of $\mathrm{Z}$ Cam during a run). The diagonal line is the value of $\log Q$ expected from scintillation noise. Two runs are badly affected by scintillation: the remainder define a curve. This curve can be explained by the bright-spot model. When $\log \langle I\rangle \lesssim 4.5$, the white dwarf is contributing little light to the system, and variations in brightness are primarily in the bright spot. If the per- 
centage of variation in the bright spot is independent of its brightness, we expect the relation between $\log Q$ and $\log \langle I\rangle$ to be a straight line parallel to the scintillation line. If $\log \langle I\rangle \gtrsim 4.6$, the white dwarf contributes most of the light of the system - the curve becomes horizontal. The curve may even go down again for $\log \langle I\rangle \gtrsim 5.0$. This might be explained by increases in the size of the disk during eruptions (as Smak (1971) has suggested occur in U Gem). The transferred matter then has less energy when it strikes the disk. The spot is fainter and the flickering decreases in amplitude.

Leung: Is your photometer a single-channel one?

Robinson: It is two-channel, but we often use it as a single-channel one.

Leung: Do you not look at a comparison star?

Robinson: We look at a comparison star at the beginning and end of each run, and take sky readings every half hour. The external errors of our brightness measurements may be a few per cent, but they are not significant on the scale of Figure 3.

Leung: If you used both channels and looked at the comparison star and the variable simultaneously, would it be much easier to sort out which is scintillation and which are real variations?

Robinson: No. Scintillation will be different for two stars in different directions in the sky. It will also be different for the two diaphragms. There's no way you could measure the light of the comparison and variable stars through the same diaphragm.

Devinney: Do I understand that the relative amount of flickering decreases at maximum light, so the light of the white dwarf is washing out the variations?

Robinson: Yes, relative to the total light of the system, the flickering goes down dramatically!

Smak: I think that your suggestion that the disk may be larger when the system is very bright is quite reasonable. Then the spot may be formed closer to the Lagrangian point and the collision velocity would be smaller than usual. Therefore, the spot would not be so bright.

Bath: How constant is the power spectrum between maximum and minimum? You said that this constancy indicates that the red star is not the source of instability, and that there is essentially no change in the mass-transfer rate during an outburst. Can you deduce from this any quantitative values of the possible range of mass-transfer rates?

Robinson: From the flickering alone, you cannot calculate the mass-transfer rate.

Bath: But you were concluding from the power spectrum that the rate must be essentially constant.

Robinson: Possibly you could determine the exact amount of change in brightness of the spot, but the observational scatter is so large that the result would probably be misleading.

Smak: I think the point is that the luminosity of the spot, as we see it in the visual region, is a fairly complicated function of the rate of mass transfer, the velocity of collision, and the physical properties of matter....

Bath: ... and therefore I don't think you can necessarily conclude that the blue star is undergoing the outburst. 
Robinson: I think you can conclude that the red star isn't erupting; because if it is, it would throw off a large amount of mass. This mass, presumably, would go towards the white dwarf, and greatly change the pattern of the flickering.

Hutchings: Would you care to comment on similar observations the Texas people have made of other stars with flickering hot spots? These spots seem to me to be a fairly important observational discovery.

Robinson: We have not yet found any inconsistency between the observations of cataclysmic variables and a hot-spot model. These systems always show flickering, and are binary systems with mass transfer. The strength of flickering varies dramatically from star to star: it is stronger in Z Cam than in most, although VV Pup flickers by almost a magnitude.

Hutchings: Does the size of the hot spot vary? Can you tell this in systems in which the hot spot is eclipsed?

Robinson: We're accumulating observations of eclipses of U Gem. We'll be able to say more about that when we have reduced them. Perhaps the size of the spot is one of the least-known parameters. The best estimate at the moment is a diameter of $3 \times 10^{9} \mathrm{~cm}$.

Thackeray: I'd like to make one 'ancient-historical' remark. As some of you may know, the first observation of the flickering of $U$ Gem was made in 1856, as I think we must believe now, with a 7-inch refractor by Norman Pogson, an assistant at the Radcliffe Observatory, Oxford. The observation was quoted by Van der Bilt (1908). I think I may claim to have seen the fluctuations of VV Pup (Thackeray et al., 1950) visually with the 74-inch reflector. I could hardly believe my eyes, but these fluctuations in intervals of a few seconds were recorded on five occasions.

Smak: I would like to say a few words about systematic differences between novae and $U$ Gem systems at minimum. The first difference was noticed long ago by Kraft and refers to the appearance of the emission lines: those in $U$ Gem systems seem stronger but of lower excitation and ionization, though there are exceptions. The second difference refers to the relative intensity of continuous radiation coming from the hot spot. As I tried to demonstrate last year at the Bamberg Colloquium, there appears to be a systematic preference among the U Gem type systems to have relatively brighter spots, though - again - there are some exceptions to this rule. Finally, I would like to show what seems to be another systematic difference between the two types of objects and it is shown in the slide (Figure 5). This diagram is basically a reproduction from an earlier paper by Kraft (1964), except that I used different symbols for novae (filled circles) and $\mathrm{U}$ Gem systems (open circles). It is a plot of rotational velocities $\left(V_{d} \sin i\right)$ against semi-amplitude $\left(K_{1}\right)$. An arbitrary line $V_{d}$ $\sin i \times K_{1}=$ const is drawn in to help show the separation. Arrows are placed at points which represent non-eclipsing systems to indicate corrections to be applied for the inclination effect. While the effect I am talking about is not very obvious, its reality seems to be supported by the case of VV Pup, which is neither a nova nor a U Gem object. It is plotted in the diagram with the $K_{1}$ value from Herbig's work and the $V_{d} \sin i$ value crudely estimated from the width of lines in his Crossley spectrograms. 


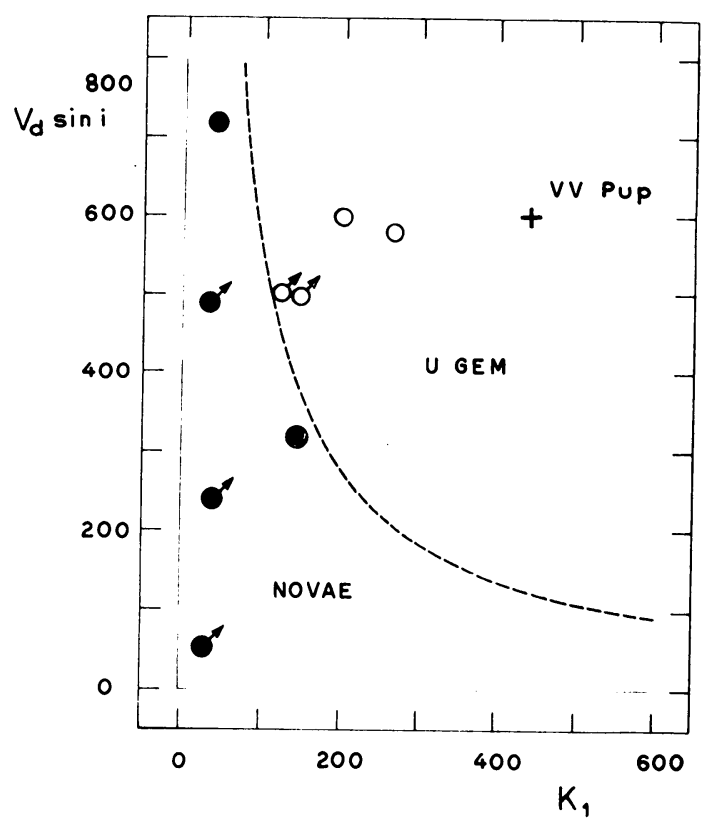

Fig. 5. Plot of emission-line width, $V_{d} \sin i$, against semi-amplitude of velocity of blue component, $K_{1}$, for novae and U Gem systems.

From the location in this diagram, it would appear then that VV Pup belongs to the U Gem group. And it is really encouraging to recall that VV Pup has the longest observed shoulder in its light curve, indicating a relatively very bright hot spot and, also, that the spectrum contains very strong hydrogen lines with no helium lines being present.

To summarize, I think we can say that there are systematic differences between novae and U Gem stars, which - in all cases - refer to the properties of the circumstellar material and in the case of the two of those, which I was talking about, they appear to reflect some systematic differences in the dynamical properties of gas streams and disks in these systems. It may well be so, therefore, that the dramatic differences in their behaviour at outbursts result, at least partly, from the different properties of the circumstellar matter.

Herczeg: You mentioned that hot spots are less important in novae than in U Gem stars. Can we perhaps say that there may be novae without hot spots? In particular, what about DQ Her in which the flickering activity seems to be much weaker than the pulsation?

Robinson: The flickering in DQ Her is very strong, and frequently hides the $71 \mathrm{~s}$ pulsation.

Herczeg: May I ask a second question? Figure 5 indicates rather clearly that radial-velocity amplitudes are systematically smaller for novae than for U Gem stars. Does this mean, perhaps, that novae have the smaller masses? 
Smak: Maybe.

Fracastoro: Does the flickering have any quasi-periodic character?

Robinson: Yes, it often looks periodic, but it is never strictly periodic. It's like any stochastic process. If you take sufficiently short runs of data, you can find very strong spikes at any frequency you choose. When you take longer runs, the spike tends to disappear.

Fracastoro: What is the sub-period of this flickering - five or ten minutes?

Robinson: The flickering varies in intervals from as short as the instrument is capable of registering up to perhaps ten minutes or more.

Kitamura: In the Elsinore meeting (Kitamura, 1970), I emphasized that the use of the Roche coordinates (with the zero-velocity curves $\xi=$ const as one of them) is useful for hydrodynamic treatment of gas motions in close binary systems. This was already pointed out by Prendergast (1960) and Kopal (1969). With the use of Roche coordinates $(\xi, \eta)$ we shall consider the case that rotating flows of inviscid gases exist around the parent star and are governed by gravitational forces only. Discussion is confined to the steady flows in the orbital plane in the case of circular orbits of the components. Taking the pressure terms into account, we start from the two scalar equations of motion on the orbital plane:

$$
\begin{aligned}
& u_{1} \frac{\partial u_{1}}{\partial \xi}+\frac{h_{1}}{h_{2}} u_{2} \frac{\partial u_{1}}{\partial \eta}+\frac{u_{1} u_{2}}{h_{2}} \frac{\partial h_{1}}{\partial \eta}-\frac{u_{2}^{2} \partial h_{2}}{h_{2} \partial \xi}-2 u_{2} h_{1}+\frac{1 \partial P}{\varrho \partial \xi}=K_{\xi}, \\
& \frac{h_{2}}{h_{1}} u_{1} \frac{\partial u_{2}}{\partial \dot{\xi}}+u_{2} \frac{\partial u_{2}}{\partial \eta}-\frac{u_{1}^{2}}{h_{1}} \frac{\partial h_{1}}{\partial \eta}+\frac{u_{1} u_{2} \partial h_{2}}{h_{1}} \partial \dot{\partial \xi}+2 u_{1} h_{2}+\frac{1 \partial P}{\varrho \partial \eta}=0 \text {, }
\end{aligned}
$$

with

$$
u_{1}=\partial \xi / \partial t \quad \text { and } \quad u_{2}=\partial \eta / \partial t
$$

where $h_{1}$ and $h_{2}$ are the metric coefficients in the transformation

$$
(\mathrm{d} x)^{2}+(\mathrm{d} y)^{2}=h_{1}^{2}(\mathrm{~d} \xi)^{2}+h_{2}^{2}(\mathrm{~d} \eta)^{2}
$$

and $q$ the mass-ratio $m_{2} / m_{1} \leqslant 1.0$ between the components. The separation of the components has been taken as the unit of length, the total mass $m_{1}+m_{2}$ as the unit of mass, and $P / 2 \pi$ as the unit of time. In doing so, the external force $K_{\xi}$ reduces to $K_{\xi}=1 /(1+q)$.

As the first approximation we have neglected the velocity components along the $\eta$-constant curves orthogonal to the zero-velocity curves, because ring-like flows would not exist very far from the parent star and, if rotating flows exist around the parent star, the velocity components along the curves $\eta=$ const should be small compared with the other components along the curves $\xi=$ const.

Thus, putting $u_{1}=0$ in the above equations of motion, and solving for $u_{2}$, we can 
have

$$
u_{2}=\frac{1}{1 \frac{\partial h_{2}}{h_{2} \partial \xi}}\left\{-h_{1}+\sqrt{h_{1}^{2}+\frac{1}{h_{2}} \frac{\partial h_{2}}{\partial \xi}\left(\frac{1}{\varrho} \partial \xi-\frac{1}{(1+q)}\right)}\right\}
$$

In this equation, the pressure term occurs in the form of $\partial P / \partial \xi$.

Assuming the adiabatic relation, it follows that

$$
\frac{1 \frac{\partial P}{\varrho} \propto-\gamma}{\gamma \xi} \frac{\partial \varrho^{\gamma-1}}{\partial \xi}, \quad(\gamma>1),
$$

where $\gamma$ denotes the ratio of specific heats.

If the gases within the ring are distributed so as to have a maximum density on the $\eta$-constant curve, we may put there

$$
\frac{\partial \varrho^{\gamma-1}}{\partial \xi}=0 \text { and so } \frac{1 \partial P}{\varrho \frac{\partial \xi}{\partial \xi}}=0 .
$$

Thus, for the density maximum within the ring we may neglect the pressure term. This is important.

Roughly speaking, the observed rotational velocity of the ring may be related to the $u_{2}$ velocity of gases at maximum density, and therefore, in the first approximation, the $u_{2}$ equation with the pressure term in it dropped may be directly compared with the observed rotational velocity. Inserting the observed rotational velocity of the ring in $u_{2}$, we can easily estimate the dimension of the ring, because the metric coefficients and their derivatives can be easily calculated as functions of the coordinates and the mass-ratio $q$.

Application to four well-known systems with gaseous rings is shown as follows:

TABLE I

Dimensions of Rings

\begin{tabular}{lccccc} 
Star & $q$ & $V_{\text {ring }}$ & $r_{\text {ring }}$ & $r_{\text {ring }} / r_{1}$ & $r_{\text {ring }} / r_{\text {Roche }}$ \\
\hline RY Gem & 0.21 & 1.32 & 0.314 & 2.62 & 0.598 \\
AW Peg & 0.16 & 1.64 & 0.242 & 1.42 & 0.439 \\
U Sge & 0.38 & 1.11 & 0.352 & 1.68 & 0.754 \\
RW Tau & 0.23 & 1.29 & 0.320 & 1.73 & 0.620 \\
\hline
\end{tabular}

In Table I, $V_{\text {ring }}$ is the observed rotational velocity of the ring expressed in the present unit; $r_{\text {ring }}, r_{1}$ and $r_{\text {Roche }}$ are the dimensions along the $y$-axis of the ring, the parent star, and the Roche limit respectively. In this computation, the data are taken from A Catalogue of Graded Photometric Studies of Close Binaries (Koch et al., 1970) and Sixth Catalogue of the Orbital Elements of Spectroscopic Binary Systems (Batten, 1967). The above result indicates that the gaseous ring is kept well inside the Roche limit. 


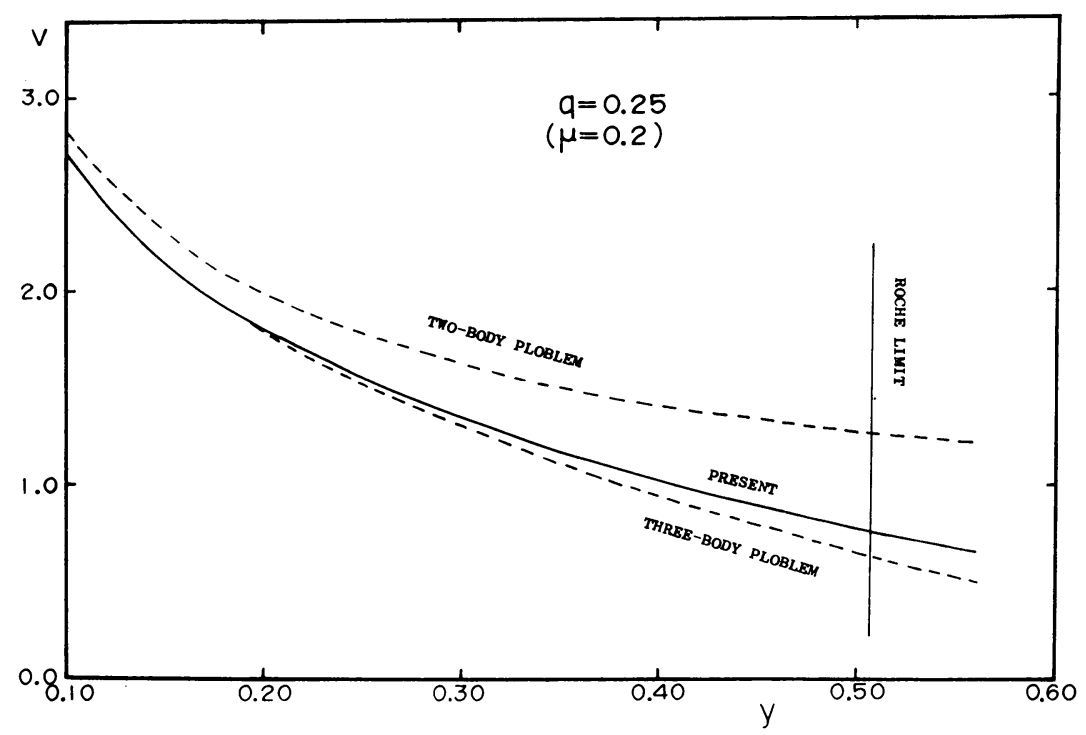

Fig. 6. Comparison of velocity $V$ at points on the $y$-axis through the primary component predicted by different approximations (see text).

Next, I would like to show you a comparison of our $u_{2}$ velocities with those from the restricted three-body problem by Huang (1967). The corresponding velocities at the same positions on the $y$-axis as used by Huang are calculated with our $u_{2}$ equation by dropping the pressure term. Figure 6 shows that the agreement is nearly perfect for the smaller $y$-values but away from the surface of the parent star the discrepancy becomes appreciable.

As the next step, we can proceed to the second approximation by taking into account the velocity component along the $\eta$-constant curves, and appropriate boundary conditions.

Biermann: I have a question about your approximations. Do you assume a disk of constant thickness, or do you make some other approximation about the thickness of flow?

Kitamura: This discussion is confined within the plane, and we need not consider the pressure term to derive a first approximation for the dimension of the ring.

Huang: The two-dimensional approximation is significant, because the gas is confined within the plane.

Biermann: But the thickness might be variable. You could treat a two-dimensional flow with variable thickness.

Kitamura: Yes, in a general treatment this should be considered.

Huang: The two-dimensional approach is allright if the $z$-component of velocity is initially very small, but not if it is initially large.

Biermann: I think you are quite right. This is a very good approximation as far as we can tell. However, there are really two quite different problems: a two-dimen- 
sional flow of constant thickness, with a central force, and a two-dimensional flow with varying thickness, in a binary system. You get a source term if the thickness varies - from the continuity equation. If the thickness changes a lot, the source term will be important. I think you could do the same kind of mathematics, including the source term, but it probably would not be important to this kind of flow unless the thickness varies a lot. It might be worthwhile to include the source term in other computations - for instance in treating the flow from the stream to the disk.

Huang: I agree with that.

R. E. Wilson: Did you make any trials to see how large a ring can be, and still look reasonably like a ring? I presume it can be no larger than the Roche lobe.

Kitamura: As Table I shows, the rings in RY Gem, AW Peg, U Sge, and RW Tau are all within the Roche lobes.

R. E. Wilson: Yes, but this is an observational result. What I meant was: have you run any purely computational trials to see how large a ring could be - without reference to the observations.

Kitamura: What I showed is that the $u_{2}$ equation enables us to estimate the size of the ring from the observed rotational velocity. From this equation alone we can say nothing about the 'purely theoretical' size of the ring.

Smak: Figure 6 shows a large difference between the results of your approximation and those of Dr. Huang's approximation. If I remember rightly, the numbers listed in Dr. Huang's paper refer to the rotating system of coordinates. If that is so, they should be corrected for the rotation of the system before being compared with the two-body case.

Bolton: The system HDE $226868=\mathrm{Cyg} \mathrm{X}-1$ is the prototype of one kind of X-ray binary characterized by X-ray variations of a factor of two or more in less than a second. There is no evidence of any periodic pulsations. The X-ray spectra of all of the known X-ray binaries are relatively flat compared to those of other X-ray sources. The identification of HDE 226868 with Cyg X-1 is now virtually certain. In the spring of 1971 a weak radio source appeared within the error box for Cyg X-1. The position of this radio source agrees with the position of the B0Ib star HDE 226868 to better than one arc second. At the same time that the radio source appeared, the X-ray source underwent a sharp decline in intensity. Thus the simultaneous $\mathrm{X}$-ray and radio variability combined with the optical-radio position agreement, is strong evidence for the identity of HDE 226868 with Cyg X-1.

Soon after the identity of Cyg X-1 and HDE 226868 was first suspected, Webster and Murdin (1972) and I (Bolton, 1972) showed that HDE 226868 was a spectroscopic binary with a period of 5.6. The velocity curve for the system is published in the references cited. The key orbital elements are as follows: $P=5.5995, K_{1}=68.2 \mathrm{~km} \mathrm{~s}^{-1}$, $e=0.09$. The velocity amplitude is large and indicates that the mass ratio cannot be more than about $3: 1$. The eccentricity is almost certainly real. The formal standard deviation of the eccentricity is 0.02 , and great care has been taken to insure that the velocities are not affected by the contamination of absorption lines by emission.

The He II $\lambda 4686$ line is seen weakly in emission on some of the spectrograms ob- 
tained at David Dunlap Observatory. The velocity derived from this line varies in antiphase to the absorption-line velocities. If the velocities derived from the He II line represent the motion of the secondary, then a mass ratio, $M_{1} / M_{2}$ of 1.5 is indicated.

Emission lines of hydrogen, and sometimes of helium, are seen between apastron and periastron but not in the other half of the period. These emission lines are not strongest at periastron as one would expect if one of the stars filled its Roche lobe. Rather the emission is strongest at a point between apastron and periastron when the separation between the two stars is decreasing most rapidly. The emission velocity indicates that material is flowing from the B0lb star toward the unseen secondary. The variation in the emission-line strengths indicates that the B0 star is losing mass to the secondary via a stellar wind.

HDE 226868 is within a degree of the center of Cyg OB3 and its radial velocity and proper motion are consistent with it being a member of the association. Its spectral type and luminosity class are also consistent with association membership, and the velocities and strengths of the interstellar $\mathrm{Ca}$ II features are very similar to those in the spectra of other association members. If HDE 226868 is a member of Cyg OB3, the distance of Cyg X-1 is approximately $2 \mathrm{kpc}$ and this implies a mass of $20 M_{\odot}$ for the B0Ib star. This places a lower limit of about $6 M_{\odot}$ for the mass of the secondary, and that mass could be as high as $14 M_{\odot}$ or $15 M_{\odot}$ : Since it appears that a collapsed stellar object is necessary in order to produce the high X-ray flux observed from this system, HDE 226868 is thus an excellent candidate for a binary system containing a black hole.

R. E. Wilson: Can you say from the rotational broadening of lines in the B-type spectrum whether the star should be rotating synchronously?

Bolton: I haven't checked that. The $V \sin i$ is fairly low - less than $100 \mathrm{~km} \mathrm{~s}^{-1}$, I think.

Herczeg: Is there any indication of an X-ray eclipse? I think someone just said one comes at the wrong phase.

Bolton: Yes, I'm afraid that I'm one of those that contributed to the impression that there is an X-ray eclipse. The early optical results by Webster and Murdin and myself only covered about a three-month period so that it was impossible to derive an accurate period for the system. There were indications in literature that there was an X-ray eclipse showing up in the high energy $(E>20 \mathrm{keV}) \mathrm{X}$-ray data. Therefore we took these data and tried to find a period within the range allowed by the optical observations that would satisfy all of the X-ray 'eclipse' points. This led to an eclipse in the wrong place.

A more careful analysis of the $\mathrm{X}$-ray results now seems to indicate that no eclipse is shown, and in any event the improved period now available from the optical data precludes the phasing together of the X-ray 'eclipse' data.

Leung: Your estimate of $20 M_{\odot}$ for the mass of a $\mathrm{B} 0 \mathrm{Ib}$ supergiant agrees with evolutionary computations by Stothers and myself (Stothers and Leung, 1971). We believe our values of masses for supergiants are quite reliable. They are in satisfactory agreement with those inferred from main-sequence turn-off. We believe we have quite 
reliable values for class Ib stars, but class Ia stars show a very wide spread in mass.

Bolton: For my purposes, it won't make much difference what mass is chosen for the supergiant. There doesn't seem to be any way that the mass of the supergiant can be forced low enough to permit the secondary to be a normal white dwarf or neutron star. Now, how high you want to put the mass is up to you.

Underhill: Class Ia certainly has a wide spread. The secondary could be a WolfRayet star - they are much less luminous than a B0 supergiant, say $-3^{\mathrm{m}} .5$ to $-4^{\mathrm{m}} .0$.

Bolton: A star with absolute magnitude -4 might be visible in the spectrum, particularly if it had emission lines. I think that we are about to hear something that tends to support your suggestion after a fashion. I have the following objection to invoking a Wolf-Rayet star or even a normal main-sequence star as the secondary even though such objects cannot be rejected on observational grounds. It does not seem possible to obtain the required X-ray fluxes from such objects without invoking unknown or ad hoc physics while the required fluxes can arise quite naturally from collapsed stars. Furthermore, if you are to say that X-rays are produced in this case by a non-collapsed star, then you must explain why other similar objects in similar situations are not X-ray sources.

Hutchings: Astronomers from the University of British Columbia and myself have been observing Cyg X-1 at Victoria, using the Image-Isocon spectrum scanner at the coudé-focus of the 48-inch telescope. The three chief advantages of this method over conventional spectrophotometry are (i) high signal-to-noise ratio, (ii) linear light response, and (iii) digital data, which enable easy and direct comparisons to be made with standard stars. We have obtained mean, rectified scans of the $\lambda 4686$ region at 8 phases through the 5.6 period. I should mention at the outset that these results are preliminary, being noisier and less carefully rectified than we hope the final results will be. The velocity changes with phase are clear, showing the $\lambda 4686$ emission feature to vary out of phase with the absorption lines. These velocities agree with Bolton's values. The $\lambda 4686$ emission is blended with $\lambda 4686$ absorption from the B star, and we do not feel there is definite evidence as yet for any variation in the strength or profile of the line.

We have further obtained the mean Cyg X-1 spectrum for the region, corrected for the primary star's orbital velocity, and the mean spectrum of the chief comparison star HD 204172. These mean spectra have a very high signal-to-noise ratio and their difference shows up as a remarkable emission-line spectrum. This must be explained either as the Cyg X-1 absorption spectrum being weakened (by a featureless continuum) or a real emission-line spectrum from the secondary. In this connection, we note that the positions of most of these lines coincide with $\mathrm{N}$ III, C III and some other weak emissions seen in similar observations of the Of stars HD 108 and HD 188001. We are working on the data further to test these conclusions. I would also like to discuss another X-ray source - Her X-1. By way of a brief introduction, the object shows three types of periodic variation in the $\mathrm{X}$-rays and two of these have been seen in the optical region. They are (i) a 36-day cycle, for 9 of which the system is active in the X-ray region of the spectrum (the optical object has no known corresponding 
behaviour); (ii) a 1-7 day cycle showing a complete, sharp X-ray eclipse lasting $\sim 0.2$ of the period. Several groups of workers have derived optical light curves which indicate a continuous light variation, covering nearly 2 mag., which shows a large $U-B$ change, and have a minimum coincident with the middle of the X-ray eclipse, (iii) a pulsation in the X-rays of period $\sim 1.24$, which shows (Tananbaum et al., 1972) a time-of-arrival periodicity (1.7) which leads to values for the orbital radius $\left(\sim 4 \times 10^{6} \mathrm{~km}\right)$ and velocity $\left(\sim 170 \mathrm{~km} \mathrm{~s}^{-1}\right)$ of the X-ray source. Very faint $(<0$ m.002) pulses have been claimed by a group at Berkeley (Davidsen et al., 1972).

David Crampton, at the Dominion Astrophysical Observatory (among others, of whom I believe Mr. Bopp is present) has obtained some dozen spectra of the object to date and these are very curious. They show a strange mixture of spectral features, some of which may be variable within hours, but whose mean behaviour suggest a change from early B-type at maximum light to late A-type at minimum. We have measured these for radial velocity and after rejecting several discrepant or poor lines, obtain a velocity curve of amplitude $\sim 50 \mathrm{~km} \mathrm{~s}^{-1}$ in antiphase with the X-ray source. We have a tentative model for your consideration. The light-curve suggests a contact or ellipsoidal type of system with a small, dark companion. The former possibility raises insoluble difficulties in regard to the non-appearance of a secondary X-ray eclipse, and for this and other reasons, we explored the possibilities of a single star distorted and heated by a small, hot companion using the light curve synthesis program of Hutchings and Hill. It is possible to reproduce the range by heating a star, at its Roche limit, by a companion of size $\sim 0.05$ or smaller. The minimum is rather broad but can be filled in by (i) small flux from the hot star (there is an ultraviolet excess which disappears at minimum light), (ii) electron-scattered light in a tenuous envelope about it, and (iii) convective heating of the very highly heated hot side. We are unable however to choose between a (cool star) polar radius 0.25 and $q$ (hot mass/cool mass) $\sim 4.0$ and a radius $\sim 0.45$ and $q<0.5$, or anywhere between these. If, however, we assume $i$ close (within $\sim 10^{\circ}$ ) to $90^{\circ}$, the duration of X-ray eclipse suggests a radius $\sim 0.4$ and $q \sim 0.5$. Using the absolute values of orbital parameters given by Tananbaum et al. (1972), we find the cool star could be an early A-type star of $2 R_{\odot}$ and $2 M_{\odot}$, making the $\mathrm{X}$-ray source $\sim 1 M_{\odot}$. From this, we expect a velocity amplitude of the visible star of $\sim 85 \mathrm{~km} \mathrm{~s}^{-1}$, but calculations of line profiles accounting for the very large heating effect show that this would appear as an observed amplitude of only $\sim 50 \mathrm{~km} \mathrm{~s}^{-1}$. The present velocity estimates, which are admittedly poor, are in keeping with this. (This is described in full in Astrophys. J. Letters, Dec. 1, 1972.)

Smak: I don't quite understand how you got that synthetic light curve. It appears to me that for almost one-half of the period, when we are looking only at the back of the large star, we should see only as much variation as results from the temperature difference between $7000 \mathrm{~K}$ and $6200 \mathrm{~K}$, unless you make convection so efficient as to transfer quite a lot of heat from the small star all round the big one.

Hutchings: You have a temperature distribution all the way over the star. I have just given extreme values. As the stars move, you see a constantly changing temperature distribution. 
Smak: I am talking about the phases when you see only the back of the large star - about 0.2 on either side of zero phase. You see only the large star - the bright one is hidden - and yet there are variations of about one magnitude, although on your model the luminosity should change by roughly only $10 \%$.

R. E. Wilson: Dr. Smak is precisely right, because I have made very much the same calculations as Dr. Hutchings has made and, in fact, the phase variation is essentially as Dr. Smak says it should be, and significantly different from that found by Dr. Hutchings. My theoretical light curve is much flatter around the time of the X-ray eclipse.

Bopp: I have some spectra of $\mathrm{HZ}$ Her (Her X-1) also. Dr. Hutchings said the type varied from $B$ to nearly $F$. This is not quite correct: the behaviour is much more complicated: the strength of the $\mathrm{K}$ line of $\mathrm{Ca}$ II varies from about $\mathrm{A} 3$ on our plates to about A7 or later around phase 0 . 9 . Absorption lines of $\mathrm{HeI}$ are always visible on our spectra, however. The strength of the helium lines varies, but the variation does not seem to be correlated with phase. In addition, blended N III and C III emission at $\lambda \lambda 4640$ to 4650 and He II emission at $\lambda 4686$ are visible on some of our plates, though the strength of these features varies in thirty minutes or less. How does Dr. Hutchings' model account for the 36-day X-ray period?

Hutchings: It doesn't! The suggestion by Tananbaum et al. (1972) of some sort of pulsation seems to me the most reasonable yet made. We looked for possible longer periodicities in our radial velocities, but have not found any yet.

Leung: Do the short-period variations originate in the small component or in the big one?

Hutchings: They are in the X-rays, so I assume they originate in the X-ray source.

Leung: If the short-period variation is associated with the 'white dwarf', it should be of the order of seconds, like the fundamental period of white dwarf.

Herczeg: Can the X-ray pulsation be observed during eclipse, or does it disappear and reappear as it should do?

Hutchings: You can see no X-rays at all duirng the eclipse.

Bolton: As I understand it, the optical observations of pulses do not always correlate with the observed X-ray pulses in the way that they should. Since the optical pulse amplitudes are so small, I would consider the whole business very shaky. The $\mathrm{X}$-ray pulsations are very regular but it is claimed that these 1.2 optical variations appear when there are no similar X-ray variations, and vice versa. The situation is not at all clear.

Oliver: I can't speak for the Lick observers, but I have spoken to those at Rochester and they did not make a very definite statement about the reality of these pulses. We have looked for the same thing at Florida. We may have detected something, but this kind of observation is extremely difficult.

Hutchings: Various observers tried at Victoria too, and found nothing stronger than $0^{\mathrm{m}} .002$.

Robinson: Synchronous photometry, which many people are doing, is very dangerous when you're trying to detect 0 m.002: almost anything can introduce 'wiggles'. You 
should obtain a long string of data, without summation, and then find the power spectrum, so you can have an idea what you're seeing at other frequencies too.

Scarfe: Does your model predict a detectable eclipse of the small object in ultraviolet light?

Hutchings: I have to check that. It certainly would give you a different light curve.

Lloyd Evans: I'd like to ask Dr. Bolton whether he can tell us anything about a rather different system which might have a black-hole secondary component. I believe Nolan Walborn has the observations.

Bolton: I believe you are referring to V453 Sco. I'm sure that Dr. Sahade could say more about this system than I can, since he has studied it spectroscopically. The system apparently consists of two stars of approximately equal surface brightness, according to the photographic photometry by Gaposchkin (1939). However, there are absorption lines visible for only one component. These lines indicate that the star that produces them is a supergiant belonging to the class of stars with exceptionally strong nitrogen lines that were defined by Walborn (1971). There are strong emission lines of hydrogen which shift in antiphase to the absorption-line spectrum. If these hydrogen lines are representative of the motion of the unseen star, then the unseen star is slightly more massive than the absorption-line object. Walborn has recently submitted a note on this system to the Astrophys. J. Letters. Shortly after that paper was submitted, I received a preprint of a paper by Shakura and Sunyaev in which they discuss the optical appearance of a black hole accreting mass through mass exchange in a binary system. Several of their predictions for a black hole accreting mass rapidly fit the observed properties of V453 Sco. These properties include a disk of high surface brightness, which produces emission lines but no absorption lines, and mass outflow from the disk. I pointed out the similarities of the observed properties of V453 Sco to the predictions of Shakura and Sunyaev, and Dr. Walborn has added a note about this to his paper.

One might expect that a black hole in V453 Sco would be a strong X-ray source. However, the disk is probably too thick in the line of sight to permit any X-rays to escape. It seems likely to me that Cyg X-1 differs in its mass-transfer rate and orbital inclination but that these two systems are fundamentally similar.

Note added in proof: Hutchings reports subsequent work on He II emission in the spectrum of Cyg X-1 in Astrophys. J. (to be published).

\section{References}

Batten, A. H.: 1967, Publ. Dominion Astrophys. Obs. 13, 119.

Bolton, C. T.: 1972, Nature 235, 271.

Davidsen, A., Henry, J. P., Middleditch, J., and Smith, H. E.: 1972, Astrophys. J. Letters 177 , L97.

Evans, D. S.: 1971, Monthly Notices Roy Astron. Soc. 154, 329.

Gaposchkin, S.: 1939, Astrophys. J. 89, 125.

Greenstein, J. L.: 1950, Publ. Astron. Soc. Pacific 62, 156.

Huang, S.-S.: 1967, Astrophys. J. 148, 793.

Johnson, M.: 1953, Observatory 73, 109. 
Joy, A. H. and Sanford, R. F.: 1926, Astrophys. J. 64, 250.

Kitamura, M.: 1970, in K. Gyldenkerne and R. M. West (eds.), Mass Loss and Evolution in Close Binaries, Copenhagen University, p. 194.

Koch, R. H., Plavec, M., and Wood, F. B.: 1970, Publ. Univ. Pennsylvania, Astron. Ser. XI.

Kopal, Z.: 1969, Astrophys. Space Sci. 5, 360.

Kraft, R. P.: 1964, Astrophys. J. 139, 457.

Kron, G. E.: 1952, Astrophys. J. 115, 301.

Lortet-Zuckermann, M. C.: 1965, Kleine Veröffentl. Remeis-Sternw. Bamberg 4, 30.

Lucy, L. B. and Solomon, P. M.: 1970, Astrophys. J. 159, 879.

Mayall, M. W.: 1937, Harvard Ann. 105, 491.

Mendoza, E. E.: 1958, Astrophys. J. 128, 207.

Moffett, T. J. and Bopp, B. W.: 1971, Astrophys. J. Letters 168, L117.

Paczyński, B.: 1967, in J. Dommanget (ed.), On the Evolution of Double Stars, p. 111 ; Commun. Obs. Roy. Belgique, Ser. B, No. 17.

Prendergast, K. H.: 1960. Astrophys. J. 132, 162.

Roxburgh, I. W. and Strittmatter, P. A.: 1965, Z. Astrophys. 63, 15.

Schild, R. E.: 1966, Astrophys. J. 146, 142.

Smak, J.: 1971, Veröffentl. Remeis-Sternw. Bamberg 9, 248.

Stothers, R. and Leung, K.C.: 1971, Astron. Astrophys. 10, 290.

Struve, O.: 1952, Publ. Astron. Soc. Pacific 64, 117.

Struve, O., Herbig, G., and Horak, H.: 1950, Astrophys. J. 112, 216.

Struve, O. and Zebergs, V.: 1959, Astrophys. J. 130, 783.

Tananbaum, H., Gursky, H., Kellogg, E. M., Levinson, R., Schreier, E., and Giacconi, R.: 1972, Astrophys. J. Letters 174, L143.

Thackeray, A. D., Wesselink, A. J., and Oosterhoff, P. Th.: 1950, Bull. Astron. Inst. Neth. 11, 193.

Van der Bilt, J.: 1908, Rech. Astron. Obs. Utrecht 3.

Von Gent, H.: 1931, Bull. Astron. Inst. Neth. 6, 99.

Walborn, N. R.: 1971, Astrophys. J. Letters 164, L67.

Walker, M. F.: 1972, Astrophys. J. 175, 89.

Warner, B. and Nather, R. E.: 1971, Monthly Notices Roy Astron. Soc. 152, 219.

Webster, B. L. and Murdin, P.: 1972, Nature 235, 37. 